Special Issue of the 8th International Advances in Applied Physics and Materials Science Congress (APMAS 2018)

\title{
Topological Transition Points in a Magnetic-Semiconductor Periodic Structure in an External Magnetic Field
}

\author{
I. Fedorin ${ }^{a, *}, \mathrm{~V} . \mathrm{FesenKO}^{b, c}, \mathrm{~V}$. Tuz ${ }^{b, c}$, G. Khrypunov ${ }^{a}$ And A. Khrypunova $^{a}$ \\ ${ }^{a}$ National Technical University "Kharkiv Polytechnic Institute", \\ Department of Materials for Electronics and Solar Cells, 61002, Kharkiv, Ukraine \\ ${ }^{b}$ International Center of Future Science, State Key Laboratory on Integrated Optoelectronics, \\ College of Electronic Science and Engineering, Jilin University, 130012, Changchun, China \\ ${ }^{c}$ Institute of Radio Astronomy of National Academy of Sciences of Ukraine, 61002, Kharkiv, Ukraine \\ In this paper iso-frequency surfaces of a ferrite-semiconductor superlattice under an action of an external \\ magnetic field are studied. The structure is considered in the subwavelength regime, when it is possible to introduce \\ effective components of permittivity and permeability tensors. It has been shown that due to the peculiarities of \\ the structure under study, a variety of topology forms arises. The topological transitions points from the open to \\ the closed forms are revealed and discussed, in particular for the cases, when iso-frequency surfaces intersect and \\ degenerate. The effective control over the topology states and topological transition points is possible by means \\ of appropriate choice of the metamaterial constituent components and their properties, like material fractions, \\ working frequency, value of the external magnetic field.
}

DOI: 10.12693/APhysPolA.135.626

PACS/topics: topology, metamaterials, photonic crystals, optical transitions, superlattice

\section{Introduction}

Recently, a lot of investigations are directed to the artificial periodical structures, metamaterials and photonic crystals, which include layers made from natural or artificial materials with spatial dispersion and different physical properties. Photonic structures can enhance the light-matter interaction by orders of magnitude, enabling the device's optical properties to be controlled externally, optically, electrically, and geometrically. Photonic structures configuration strongly influence the elementary excitations, which make it possible to tailor the spectra of elementary excitations. Such structures provide new opportunities for engineering the media, which could allow additional control over the properties of propagating waves in the millimeter, terahertz, and optical ranges [1-2].

Generally, there are several physical characteristics that are usually used to describe physical properties of photonic systems, such as frequency, wave vector, polarization, and phase. Over the past few years, the new indispensable physical characteristic of the photonic system has emerged, namely the topology of the photonic systems. In a narrow sense the photonic crystal topology is a property of a photonic material that characterizes the quantized global behavior of the wave functions on its entire dispersion band. Just as the topology of the Fermi surface defines the properties of the free electrons in metals and semiconductors, the geometry of the isofrequency surface in the phase space of the propagating

*corresponding author; e-mail: fedorin.ilya@gmail.com electromagnetic waves determines the optical properties of the corresponding optical materials. Thus, the study of the photonic crystal topology behavior may open a possibility to discover fundamentally new states of light with unusual properties and new application opportunities throughout photonics, like the photonic analog of the topological insulators [3-5].

An unusual class of subwavelength photonic structures, which exhibit an optical topological transition (OTT) from a standard closed ellipsoid dispersion, which is usual for isotropic (with spherical iso-surface) and anisotropic (with spherical and ellipsoidal iso-surfaces) dielectrics with positive material parameters $\mu$ and $\varepsilon$, to the open hyperboloid dispersion, that occurs in metamaterials, composed from $\mu$-negative and/or $\varepsilon$-negative materials, has been recently studied by a number of researchers. Naturally, being induced by the changes of the average electromagnetic response, such topological transitions can be understood in the long-wave limit, which ignores the finite size of the constituent materials unit cells. The fact that hyperbolicity requires metallic behavior in one direction and insulating behavior in the other direction leads to the requirement that metals, semiconductors, or ferrites along with dielectrics must be used as building blocks of such structures [6-8].

There is a conventional classification for anisotropic hyperbolic media with different topological phases. In a uniaxial case, type I hyperbolic structures have one component of the principal permittivity or permeability tensor component negative, and its hyperboloid surface are two sheeted. In contrast, type II structures have two components of principal permittivity or permeability negative and single sheeted hyperboloid surface. The type II structure is highly reflective as it is more metal- 
lic. In a general case of biaxial hyperbolic crystal, where all three principal permittivity or permeability tensor components are different and one component is negative, the iso-frequency surface for the extraordinary ray is an asymmetric hyperboloid. The changes in topology from an ellipsoid to a hyperboloid are responsible for many of the important properties of these materials [9-10]. As it was recently shown, the characteristic frequencies of gyroelectric and gyromagnetic materials, used as building blocks of artificial structures, can be closely spaced within the same frequency band, which may result in a number of new OTT phenomena [11-13].

Therefore, combining together these two different classes of materials within a single structure results in the fact that the electromagnetic plane waves can exhibit new kinds of wave-vector surfaces which brings unusual physical phenomena and possibility of new applications which is the subject of the present study. In the present work, we focus on the fundamental concept and the general properties of non-local optical topological transitions in the gyroelectromagnetic structure composed from gyroelectric and gyromagnetic materials and are under an action of an external magnetic field, which can give a deep insight into the physical properties of such materials, such as the positive and negative reflection or refraction, clarify the diffraction divergence of electromagnetic waves, to name but a few. By properly combining and tuning the constituent parameters of gyroelectromagnetic structure under study, the complex iso-frequency surfaces, which represent a combination of hyperboloid, elliptical, and spheroid topological surfaces, can be obtained. The results obtained in the paper may potentially lead to an entire new class of OTT's, not found within the conventional effective media.

\section{Geometry of the problem and basic equations}

Further, we are interested in the iso-frequency surfaces (surfaces of wave vectors) of a multilayered periodic structure infinitely extended along the $y$-axis. It consists of alternating magnetic (with constitutive parameters $\mu_{m}, \varepsilon_{m}$ ) and semiconductor (with constitutive parameters $\left.\mu_{s}, \varepsilon_{s}\right)$ layers having thicknesses $d_{m}$ and $d_{s}$, respectively, whereas the structure period is $d=d_{m}+d_{s}$. It is assumed that the magnetic and semiconductor layers are magnetized uniformly by an external static magnetic field $\boldsymbol{M}$ which is aligned along the $z$-axis transversely to the structure periodicity as seen in Fig. 1.

It is supposed that all characteristic dimensions, namely the thicknesses of the magnetic and semiconductor layers as well as the period of the given structure satisfy the long-wavelength limit, i.e. they are all much smaller than the wavelength in the corresponding part of the composite medium $\left(d_{m} \ll \lambda, d_{s} \ll\right.$ $\lambda, d \ll \lambda)$, and, thus, the multilayered structure is considered to be a finely-stratified one. In view of this assumption, the standard homogenization procedure from the effective medium theory is applied in or-

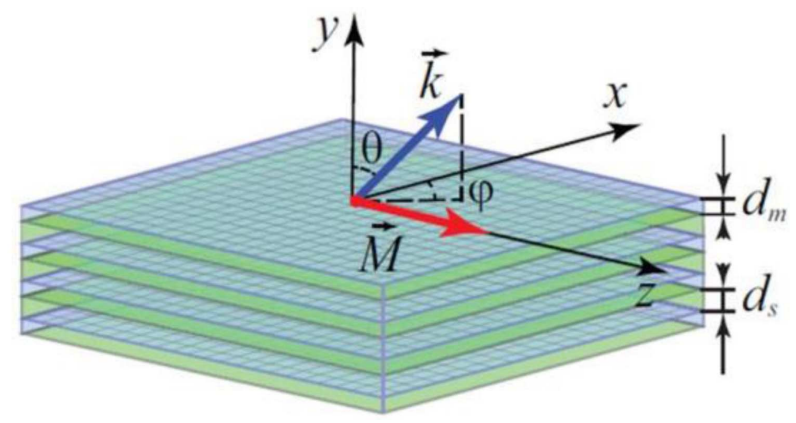

Fig. 1. Geometry of the problem under study.

der to derive averaged expressions for effective constitutive parameters of the multilayered structure. Therefore, the whole structure is approximated as an infinite gyrotropic space, which is then characterized by relative effective permeability $\mu_{\text {eff }}$ and relative effective permittivity $\varepsilon_{\text {eff }}$ represented as the second-rank tensors $\mu_{e f f}=\left[\mu_{x x}, \mu_{x y}, 0 ;-\mu_{x y}, \mu_{y y}, 0 ; 0,0, \mu_{z z}\right]$ and $\varepsilon_{e f f}=\left[\varepsilon_{x x}, \varepsilon_{x y}, 0 ;-\varepsilon_{x y}, \varepsilon_{y y}, 0 ; 0,0, \varepsilon_{z z}\right]$, whose expressions are derived via underlying constitutive parameters of magnetic $\left(\mu_{m}, \varepsilon_{m}\right)$ and semiconductor $\left(\mu_{s}, \varepsilon_{s}\right)$ layers one can find in $[13,14]$.

From Maxwell's equations, the bi-quadratic dispersion equation between the frequency $\omega$ and wavevector $\boldsymbol{k}=k(\sin \theta \cos \varphi, \sin \theta \sin \varphi, \cos \theta)$ for the electromagnetic waves in the infinite gyroelectromagnetic medium can be written as follows:

$$
\begin{aligned}
& \frac{k^{4}}{k_{0}^{4}} \overline{\varepsilon \mu}\left(\frac{\sin ^{2} \theta}{\varepsilon_{z z}}+\frac{\cos ^{2} \theta}{\bar{\varepsilon}}\right)\left(\frac{\sin ^{2} \theta}{\mu_{z z}}+\frac{\cos ^{2} \theta}{\bar{\mu}}\right) \\
& -\frac{k^{2}}{k_{0}^{2}}\left[\left(\varepsilon_{x x} \mu_{y y}+\varepsilon_{y y} \mu_{x x}-2 \varepsilon_{x y} \mu_{x y}\right) \cos ^{2} \theta\right. \\
& \left.+\left(\bar{\mu} \frac{\varepsilon_{\perp}}{\varepsilon_{z z}}+\bar{\varepsilon} \frac{\mu_{\perp}}{\mu_{z z}}\right) \sin ^{2} \theta\right]+\varepsilon_{\perp} \mu_{\perp}=0,
\end{aligned}
$$

where $k_{0}=\omega / c, c$ is the speed of light in the vacuum, and $\bar{\varepsilon}=\varepsilon_{x x} \cos ^{2} \varphi+\varepsilon_{y y} \sin ^{2} \varphi, \varepsilon_{\perp}=\varepsilon_{x x} \varepsilon_{y y}+\varepsilon_{x y}^{2}$, $\bar{\mu}=\mu_{x x} \cos ^{2} \varphi+\mu_{y y} \sin ^{2} \varphi, \mu_{\perp}=\mu_{x x} \mu_{y y}+\mu_{x y}^{2}$ are introduced as generalized effective constitutive parameters. Equation (1) describes a two-sheeted dispersion surface in $\boldsymbol{k}$ space known as iso-frequency surface or refractive index surface.

\section{Topological transitions in iso-frequency surfaces of a gyroelectromagnetic structure}

Let us consider the iso-frequency surface (surface of wave vectors) of electromagnetic waves propagating in an unbounded gyroelectromagnetic structure, using the typical constituent parameters which are inherent to available materials (here we follow the results of [12] where a magnetic-semiconductor composite in the form of a barium-cobalt/doped-silicon superlattice is considered) we perform our calculations for the microwave band, albeit all results can be easily extrapolated to other parts 
of spectrum. The characteristic resonant frequencies of the underlying magnetic and semiconductor materials of the superlattice appear to be different but rather closely spaced within the same frequency band.

The topological transition points for the structure under study, where the iso-frequency surface changes between closed and open geometries are fully described by an effective permittivity $\varepsilon_{\text {eff }}$ and relative effective permeability $\mu_{e f f}$ tensors components.

For numerical calculations, hereafter, for the magnetic constitutive layers, under saturation magnetization of $M=2000 \mathrm{G}$, parameters are: $f_{0}=\omega_{0} / 2 \pi=4.2 \mathrm{GHz}$, $f_{m}=\omega_{m} / 2 \pi=8.2 \mathrm{GHz}, b=0, \varepsilon_{m}=5.5$; for the semiconductor constitutive layers, parameters are: $f_{p}=\omega_{p} / 2 \pi=10.5 \mathrm{GHz}, f_{c}=\omega_{c} / 2 \pi=9.5 \mathrm{GHz}$, $\nu=0, \varepsilon_{l}=1.0, \mu_{s}=1.0$. The free space wave number $k_{0}=155.5 \mathrm{~m}^{-1}$. For theoretical study we neglect the losses in the structure that is we assume that electron collision frequency in the semiconductor as well as magnetic damping in the magnetic layer are zero.
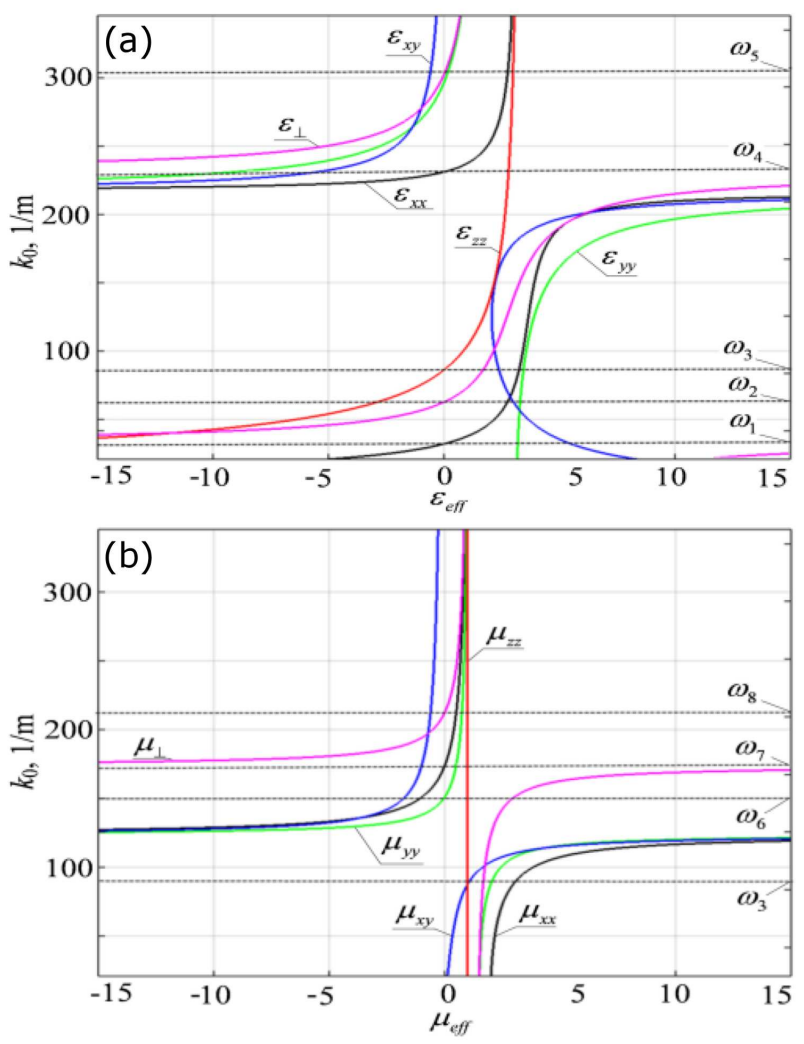

Fig. 2. Effective permittivity (a) and permeability (b) as a function of free space wave number $k_{0}$.

Since the material response and iso-frequency contours are fully described by an effective permittivity $\varepsilon_{\text {eff }}$ and effective permeability $\mu_{\text {eff }}$ tensors components, let us consider the corresponding frequency dependences (in terms of free space wave number) at $\delta_{1}=\delta_{2}=0.5$, which is presented in Fig. 2. The dotted lines denote the topological transition points where the iso-frequency surface changes between closed and open geometries. The topological iso-frequency surfaces in different frequency ranges are presented in Fig. 3. We can distinguish eight frequencies at which OTT's occur.

I region $\left(\omega<\omega_{1}\right)$. Principal effective permeability components are positive, while $\varepsilon_{\perp}>0, \varepsilon_{x x}<0, \varepsilon_{y y}>0$ and $\varepsilon_{z z}<0$. In this case, the topological transition occurs at the frequency $\omega_{1}$, where $\varepsilon_{x x}=0$. In region I, the single sheeted hyperboloid infinitely extends along the $y$-axis for extraordinary wave, i.e. along the periodicity axis, and there is no propagation for ordinary wave (empty surface), due to the pure imaginary values of ordinary wave vector component.

II region $\left(\omega_{1}<\omega<\omega_{2}\right)$. Principal effective permeability components are positive, while $\varepsilon_{\perp}<0, \varepsilon_{x x}>0$, $\varepsilon_{y y}>0$ and $\varepsilon_{z z}<0$. Topological transition occurs at the frequency $\omega_{2}$, where $\varepsilon_{\perp}=0$. In region II double sheeted hyperboloid infinitely extends along the $z$-axis for ordinary wave, i.e. parallel to the direction of an external magnetic field, and there is no propagation for extraordinary wave. Remarkably, there are no propagating waves either extraordinary or ordinary polarized in the cross-section of the iso-frequency surface by the coordinate plane $k_{z}=0$, due to pure imaginary wave vector components.

III region $\left(\omega_{2}<\omega<\omega_{3}\right)$. Principal effective permeability components are positive, while $\varepsilon_{\perp}>0, \varepsilon_{x x}>0$, $\varepsilon_{y y}>0$ and $\varepsilon_{z z}<0$. Topological transition occurs at the frequency $\omega_{3}$, where $\varepsilon_{z z}=0$. In region III the transition to the closed ellipsoidal dispersion surface for extraordinary wave occurs, while for ordinary waves there is still double sheeted hyperboloid infinitely extended along the $z$-axis.

IV region $\left(\omega_{3}<\omega<\omega_{6}\right)$. Principal effective permittivity components are positive, while $\mu_{\perp}>0, \mu_{x x}<0$, and $\mu_{y y}<0$. Topological transition occurs at the frequency $\omega_{6}$, where $\mu_{y y}=0$. In region IV there are two closed iso-frequency surfaces for both waves which intersect. In the points of intersection the dispersion surface degeneracy takes place, and the two independent modes no more exist. These points, also called diabolic points, are points of a double eigenvalue with two linearly independent eigenvectors (the optical axes of the crystal). It is known that at such points a number of interesting physical phenomenon occur, like conical refraction or surface states existence [15-17].

$\mathrm{V}$ region $\left(\omega_{6}<\omega<\omega_{7}\right)$. Principal effective permittivity components are positive, while $\mu_{\perp}>0, \mu_{x x}<0$, and $\mu_{y y}>0$. Topological transition occurs at the frequency $\omega_{7}$, where $\mu_{x x}=0$. The iso-frequency surface for extraordinary waves is closed ellipsoid, while for ordinary waves it changes from the closed ellipsoid to the open two sheeted hyperboloid extended along $x$-axis.

VI region $\left(\omega_{7}<\omega<\omega_{8}\right)$. Principal effective permittivity components are positive, while $\mu_{\perp}<0, \mu_{x x}>0$, and $\mu_{y y}>0$. Topological transition occurs at the frequency $\omega_{8}$, where $\mu_{\perp}=0$. In this case there are no propagating ordinary polarized waves, while the iso-frequency surface for extraordinary waves is closed ellipsoid. 

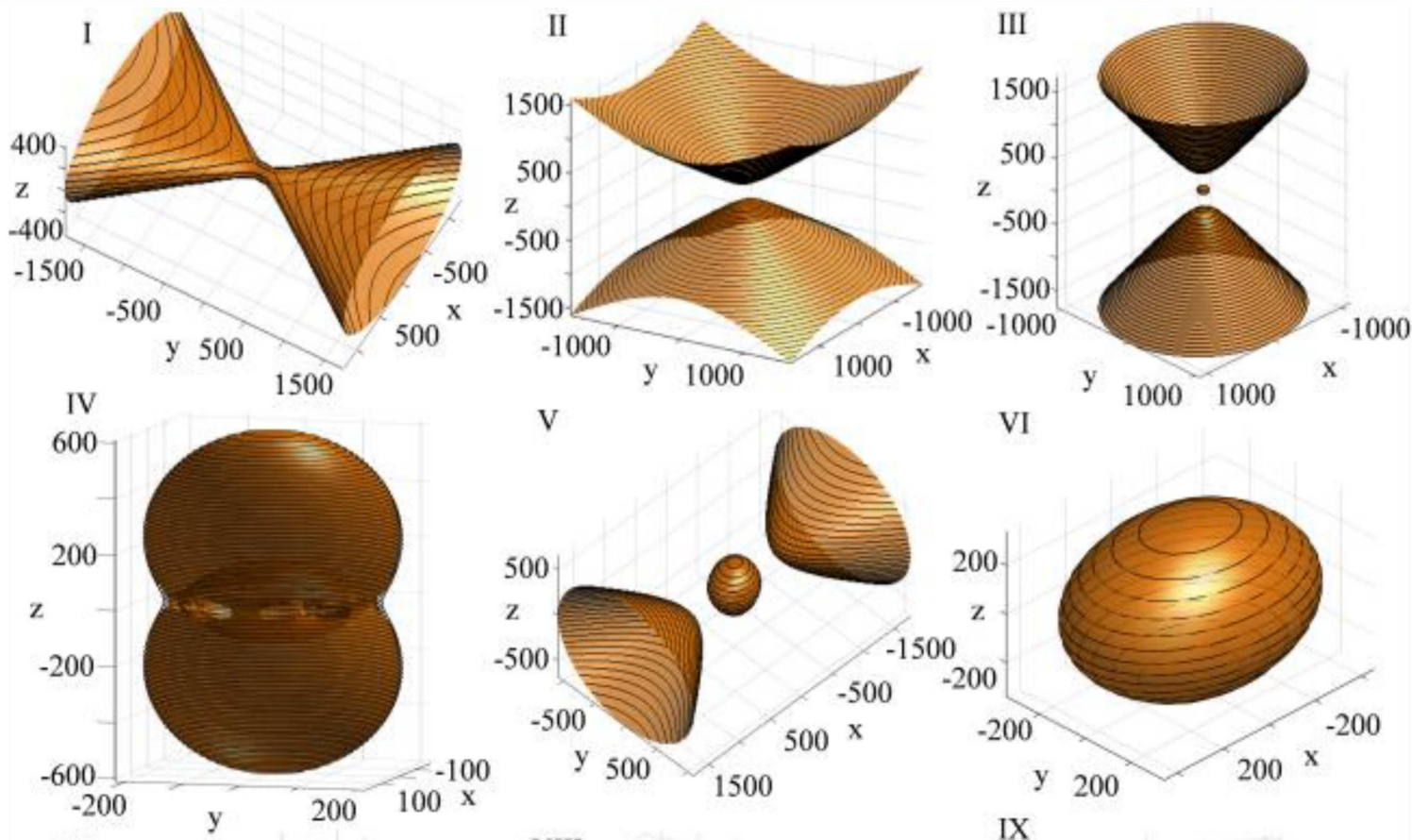

VI
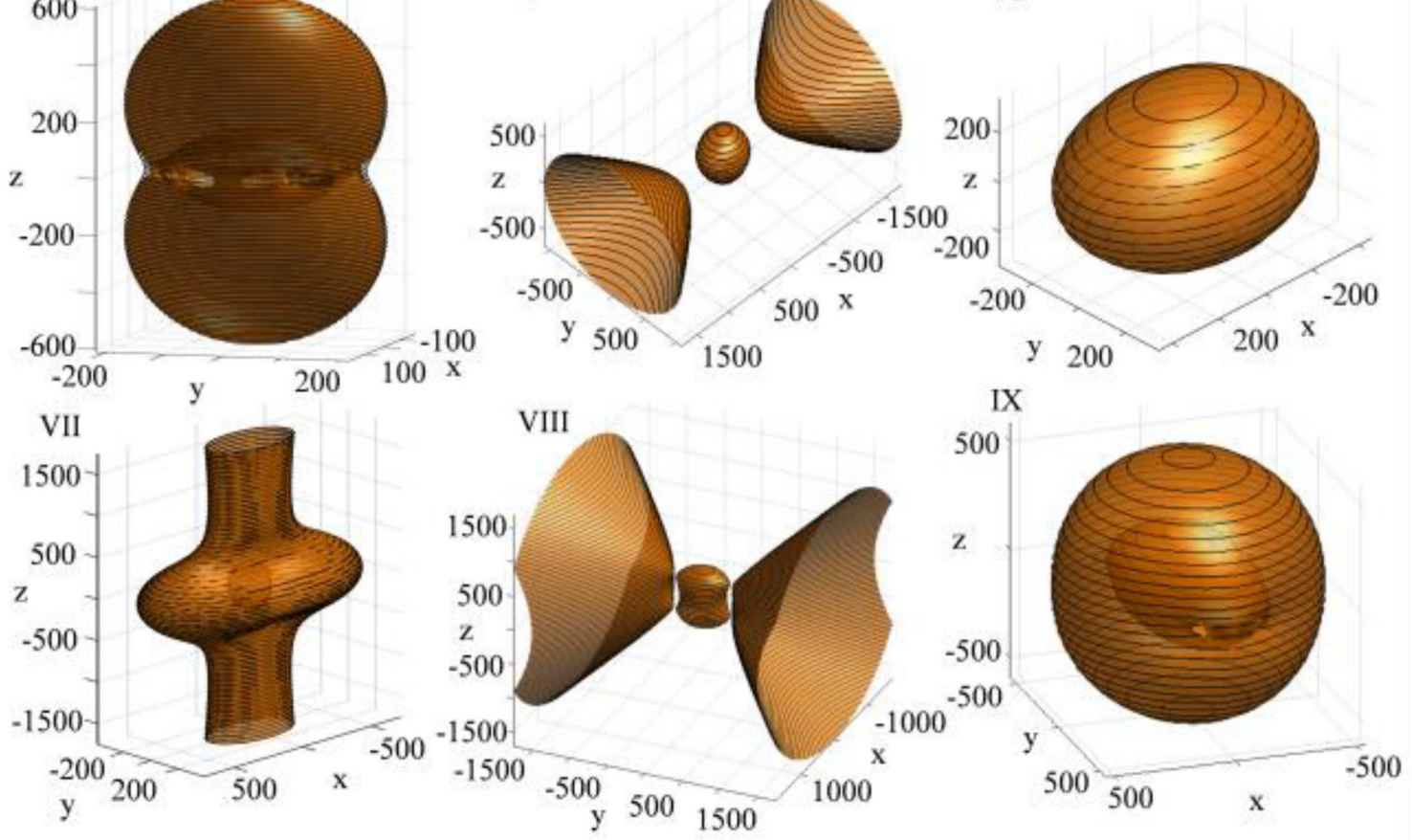

Fig. 3. Iso-frequency surfaces of the gyroelectromagnetic structure under study in the different frequency ranges.

VII region $\left(\omega_{8}<\omega<\omega_{4}\right)$. Principal effective permeability components are positive, while $\varepsilon_{\perp}>0, \varepsilon_{x x}<0$, $\varepsilon_{y y}<0$, and $\varepsilon_{z z}>0$. Topological transition occurs at the frequency $\omega_{4}$, where $\varepsilon_{x x}=0$. The isofrequency surface for ordinary waves is closed ellipsoid, while for extraordinary waves it represents complex surface which is a combination of closed ellipsoid and open surfaces [18].

VIII region $\left(\omega_{4}<\omega<\omega_{5}\right)$. Principal effective permeability components are positive, while $\varepsilon_{\perp}<0, \varepsilon_{x x}>0$, $\varepsilon_{y y}<0$, and $\varepsilon_{z z}>0$. Topological transition occurs at the frequency $\omega_{5}$, where $\varepsilon_{\perp}=0$. In this range the iso-frequency surface for ordinary waves remains closed ellipsoid, while the iso-frequency surface for extraordinary waves possess topological transition to the open two-sheeted hyperboloid, infinitely extended along the $y$ axis. Remarkably, the hyperboloid and ellipsoid intersect in four points, where the topology degeneracy occurs.

IX region $\left(\omega>\omega_{5}\right)$. All principal effective permeability and effective permittivity components are positive. In this case the iso-frequency surfaces for both waves are standard type closed ellipsoids, which intersect at four points. At these intersection points the topology singularity occurs, where the conical refraction phenomenon can take place under the certain conditions.

Let us now consider the topological transitions for different metamaterial filling factors $\left(k_{0}=155.5 \mathrm{~m}^{-1}\right)$. The corresponding permittivity and permeability dependences are presented in Fig. 4.

Under the chosen material parameters the change in the superlattice geometric factors $\delta_{m}$ and $\delta_{s}$ is mainly responsible for topological transitions in the iso-frequency surface of the ordinary waves, while the iso-frequency surface of extraordinary waves remains the closed ellipsoid regardless of the $\delta_{m}$ or $\delta_{s}$ values. The latter is due to the fact that $\mu_{z z}, \varepsilon_{\perp}, \varepsilon_{x x}$, and $\varepsilon_{y y}$ are positive over the entire range of filling factor values. It should be noted that when $\delta_{m}=0$ the structure represents a bulk semiconductor with ellipsoid iso-frequency surface for extraordinary waves, whereas when $\delta_{m}=1$ the structure represents a bulk ferrite and the iso-frequency surface for extraordinary waves is a sphere with radius $k_{0} \sqrt{\varepsilon_{m}}$. 

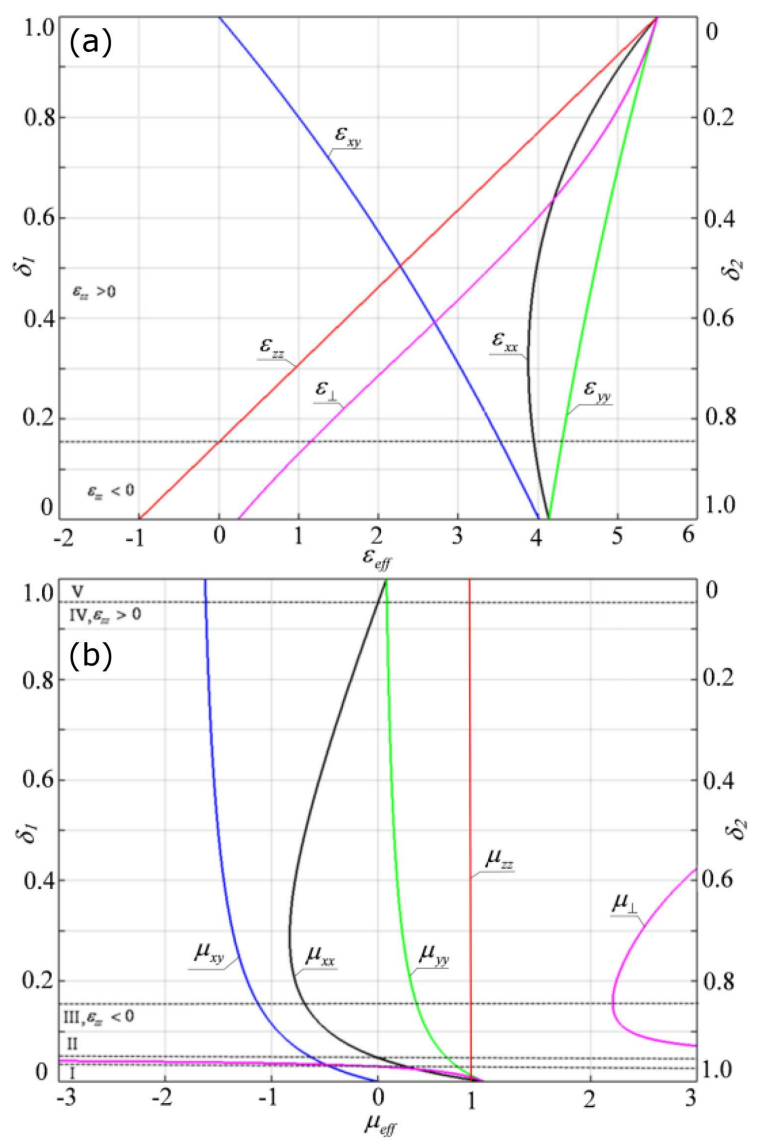

Fig. 4. Effective permittivity (a) and permeability (b) as a function of geometrical factors of magnetic $\delta_{1}$ and semiconductor $\delta_{2}$ materials.

On the other hand, the iso-frequency surface for ordinary waves undergoes a number of extreme topological transitions depending on the values of $\delta_{m} / \delta_{s}$. These regions are defined by the values where $\mu_{\perp}, \mu_{x x}, \varepsilon_{z z}$ and/or $\mu_{y y}$ change the sign. Let us note that $\mu_{y y}$ is positive over the whole range of interest. We can distinguish five bands of interest: I. $\mu_{\perp}>0, \mu_{x x}>0$, and $\varepsilon_{z z}<0$, II. $\mu_{\perp}<0$, $\mu_{x x}>0$, and $\varepsilon_{z z}<0$, III. $\mu_{\perp}<0, \mu_{x x}<0$, and $\varepsilon_{z z}<0$, IV. $\mu_{\perp}<0, \mu_{x x}<0$, and $\varepsilon_{z z}>0$, V. $\mu_{\perp}<0, \mu_{x x}>0$, and $\varepsilon_{z z}>0$.

In band I, the iso-frequency surface for ordinary waves is open two-sheeted hyperboloid, since components $\mu_{\perp}$, $\mu_{x x}$, and $\varepsilon_{z z}$ have opposite signs. This hyperboloid infinitely extends along the $z$-axis.

In band II, where both $\mu_{\perp}$ and $\varepsilon_{z z}$ are negative, whereas $\mu_{x x}$ and $\mu_{y y}$ are positive, the two-sheeted hyperboloid surface gradually closes and turns into the onesheeted hyperboloid extended infinitely along the $z$-axis. Moreover, this hyperboloid under the certain material parameters intersects with the ellipsoid, which describes the propagation of the extraordinary waves at four points, so that the diabolic points occur.

In band III, where $\mu_{\perp}, \varepsilon_{z z}$, and $\mu_{x x}$ are negative, the iso-frequency surface for ordinary waves is degenerated into a two single-sheeted hyperboloids which revolution axes are perpendicular to each other. In this case the influence of structure hyperbolicity associated with the direction of an external magnetic field ( $z$-axis) as well as the hyperbolicity associated with the direction of the periodicity of the structure along $y$-axis occurs. As a result, a new unique topological surface, which can be named as bi-hyperbolic takes place [19].

In band IV, where component $\mu_{\perp}$ is negative, while $\varepsilon_{z z}$ is positive, but $\mu_{x x}$ and $\mu_{y y}$ have different sings (negative and positive, respectively). The iso-frequency surface for ordinary waves is also an open form. Although, in contrast to band I, in this case the iso-frequency surface is a double-sheeted hyperboloid surface, which extends along the $y$-axis.

Finally, in band $\mathrm{V}$, where $\mu_{\perp}$ is negative, while $\varepsilon_{z z}$, $\mu_{x x}$, and $\mu_{y y}$ are positive, the structure shows metallic behavior with no propagating waves of the given polarization. In this case the wave vector of the ordinary wave acquires pure imaginary values.

\section{Conclusion}

We have studied the topological transition points in iso-frequency surfaces of an artificial gyroelectromagnetic structure. The gyroelectromagnetic structure represents the magnetic-semiconductor superlattice under an action of an external magnetic field. The system is considered in the subwavelength approximation. The effective medium theory is applied to achieve the homogenized parameters of the system. It is demonstrated that rich diversity of topology forms, like conical singularity or diabolic points, combination of different open and closed form surfaces, can be achieved by properly choosing the parameters of the constituent materials. The topological transition points were found and described. It is shown that the topological transitions can be reconfigurable by simply adjusting the material fraction, physical, and geometrical parameters of the system.

\section{References}

[1] J.D. Joannopoulos, S.G. Johnson, J.N. Winn, R.D. Meade, Photonic Crystals: Molding the Flow of Light, 2nd ed., Princeton University Press, New Jersey 2008.

[2] F. Bass, A. Bulgakov, Kinetic and Electrodynamic Phenomena in Classical and Quantum Semiconductor Superlattices, Nova Sci. Publ., New York 1997.

[3] S. Ishii, E. Narimanov, Nat. Photon. 5, 17824 (2015).

[4] L. Lu, J.D. Joannopoulos, M. Soljacic, Nat. Photon. 8, 821 (2014).

[5] M. Mencagli, C.D. Giovampaola, S. Maci, IEEE Trans. Antennas Propag. 64, 2319 (2016).

[6] P. Shekhar, J. Atkinson, Z. Jacob, Nano Converg. 1, 14 (2014).

[7] E.E. Narimanov, A.V. Kildishev, Nat. Photon. 9 214 (2015).

[8] I. Fedorin, Prog. Electromagn. Res. B 67, 17 (2016). 
[9] S.S. Kruk, Z.J. Wong, E. Pshenay-Severin, K. O'Brien, D.N. Neshev, Y.S. Kivshar, X. Zhang, Nat. Commun. 7, 11329 (2016).

[10] W. Li, Z. Liu, X. Zhang, X. Jiang, Appl. Phys. Lett. 100, 161108 (2012)

[11] I. Fedorin, V. Baibak, A. Bulgakov, Eur. Phys. J. Appl. Phys. 66, 20502 (2014).

[12] R.X. Wu, T. Zhao, J.Q. Xiao, J. Phys. Condens. Matter. 19, 026211 (2007).

[13] V.R. Tuz, V.I. Fesenko, I.V. Fedorin, H.B. Sun, V.M. Shulga, Superlatt. Microstruct. 103, 285 (2017).
[14] I. Fedorin, A. Bulgakov, in: Proc. 42nd Europ. Microwave Conf. (EuMC), Amsterdam 2012, p. 1229.

[15] K.E. Ballantine, J.F. Donegan, P.R. Eastham, Phys. Rev. A 90, 013803 (2014).

[16] A.A. Bulgakov, I.V. Fedorin, Opt. Spectrosc. 112, $474(2012)$.

[17] S.H. Nam, A.J. Taylor, A. Efimov, Opt. Expr. 18, 10120 (2010).

[18] V.R. Tuz, I.V. Fedorin, V.I. Fesenko, Opt. Lett. 42, 4561 (2017). 